\title{
Análisis de la calidad nutritiva forestal en el noroeste de la Península Ibérica
}

\section{Analysis of the nutritive forest quality in the northwest of the Iberian Peninsula}

\author{
González-Hernández, M.P., Mosquera-Losada, M.R., Romero, R., Rigueiro, A. \\ Departamento de Producción Vegetal y Proyectos de Ingeniería. Escuela Politécnica superior. \\ Universidad de Santiago de Compostela. 2700- Lugo \\ *Autor para correspondencia: pilar.gonzalez@usc.es
}

\section{Resumen}

El conocimiento de la calidad nutritiva de la vegetación es esencial para el desarrollo de una gestión eficiente y ecológicamente sostenible de los sistemas silvopastorales. Se compararon 11 parámetros nutricionales: Fibra Ácido Detergente (FAD), lignina, celulosa, sílice, proteína bruta (PB), digestibilidad in vitro (DMO), materia seca (MS), fósforo $(\mathrm{P})$, calcio $(\mathrm{Ca})$, magnesio $(\mathrm{Mg})$ y potasio $(\mathrm{K})$ en 47 especies vegetales presentes en robledales, pinares y matorrales gallegos. Para facilitar la comparativa de la calidad nutritiva entre las diferentes formaciones forestales se utilizó el Análisis de Componentes Principales (ACP), técnica de análisis multivariante que permite la interpretación de las relaciones (distancia) entre un número elevado de especies definidas por una variedad de parámetros nutricionales. La representación gráfica de los dos primeros componentes evidenció una agrupación coherente de las formaciones forestales, separando especies típicas de tojales y brezales de otras propias del sotobosque de robledales. El primer eje (CP1) explicó un 50\% de la variabilidad de los datos, situándose en su lado positivo las especies con una mayor digestibilidad y un más alto contenido en K. Su lado opuesto agrupó a especies de las familias Ericaceae, Cistaceae y del género Ulex que presentaron mayor contenido en celulosa y en MS. El segundo componente (CP2), representado por el Ca y lignina, explicó un 16\% de la varian$\mathrm{za}$, contribuyendo de manera limitada a una ordenación concluyente de las especies.

\section{Summary}

The knowledge of the nutritive quality of the vegetation is essential for the development of an efficient and ecologically sustainable management of silvopastoral systems. 11 nutritional parameters were compared: Acid Fiber Detergent (FAD), lignin, cellulose, silica, crude protein (PB), in vitro digestibility (BMD), dry matter $(\mathrm{DM})$, phosphorus $(\mathrm{P})$, calcium $(\mathrm{Ca})$, magnesium $(\mathrm{Mg})$ and potassium $(\mathrm{K})$ in 47 plant species present in oak groves, pine groves and Galician scrub. To facilitate the comparison of the nutritional quality between the different forest formations, Principal Component Analysis (PCA) was used, a multivariate analysis technique that allows the interpretation of relationships (distance) among a large number of species defined by a variety of nutritional parameters The graphic representation of the first two components showed a coherent grouping of forest formations, separating typical species of Ulex formations and heaths from others typical of oak groves. The first axis (PC1) explained 50\% of the variability of the data, with the positive side of the species with greater digestibility and a higher content of K. Its opposite side grouped species of the families Ericaceae, Cistaceae and Ulex genus that presented higher content in cellulose and DM. The second component (CP2), represented by $\mathrm{Ca}$ and lignin, explained $16 \%$ of the variance, contributing in a limited way to a conclusive ordering of the species.

Palabras clave: análisis multivariante, contenido mineral, digestibilidad in vitro, fibra ácido detergente, Galicia, proteína bruta.

Keywords: multivariate analysis, mineral content, in vitro digestibility, acid detergent fiber, Galicia, crude protein. 


\section{Introducción}

El conocimiento de la calidad nutritiva de las especies vegetales es esencial para evaluar los recursos alimenticios disponibles en los sistemas de pastoreo, ya que éstos vienen determinados por las características nutricionales de las especies presentes y de la proporción relativa de las mismas (Van Soest, 1982; GonzálezHernández y Silva-Pando, 1999). Una evaluación holística de la calidad nutritiva de las comunidades vegetales (entendida ésta como los recursos nutricionales existentes, independientemente de la especie animal en pastoreo) integra múltiples parámetros nutricionales $\mathrm{y}$, a menudo, también un número amplio de especies. Entre los parámetros que definen la calidad nutritiva de la vegetación se encuentran la cantidad de proteína bruta (PB), el porcentaje de digestibilidad in vitro (DMO), la materia seca (MS), el contenido mineral (fósforo (P), calcio (Ca), magnesio $(\mathrm{Mg})$ y potasio $(\mathrm{K})$ ), y el porcentaje de fibra (Fibra Ácido Detergente (FAD), en sus distintas fracciones lignina, celulosa, sílice). La digestibilidad y los niveles de proteína bruta de los forrajes se relacionan con una mayor calidad, mientras que la fibra suele estar asociada negativamente con ésta (Van Soest, 1982). Por otro lado, concentraciones inadecuadas de minerales en el pasto pueden afectar negativamente a la salud del animal, ya que éstos desempeñan funciones estructurales, actúan sobre el equilibrio ácido-base, además de participar en reacciones enzimáticas del metabolismo.

En estudios previos sobre la calidad nutritiva de los recursos disponibles para pastoreo en comunidades forestales de Galicia (González-Hernández y SilvaPando, 1999; González-Hernández et al., 2000), ya se apunta la diferencia existente entre la calidad nutritiva del sotobosque de robledales, pinares y monocultivos de eucalipto, así como entre diversas comunidades de matorral (xestas, tojales y brezal). Sin embargo, una interpretación más pormenorizada de los datos de dichos estudios se ve dificultada por el número elevado de parámetros nutricionales que definen a la calidad nutritiva y las numerosas especies que constituyen las comunidades vegetales estudiadas.

En este contexto, las técnicas de análisis multivariante pueden ser una herramienta útil al facilitar la interpretación de las relaciones (distancia) entre un número elevado de especies definidas por una variedad amplia de parámetros nutricionales. Dentro de este tipo de técnicas multivariantes, diversos estudios han validado la eficiencia del análisis de componentes principales (ACP) al permitir una organización gráfica de las especies en base a la similitud y a las diferencias de sus características nutricionales (Hejcman et al., 2014; Hejcmanová et al., 2014; González-Hernández et al., 2016; Hejcman et al., 2016). El principal objetivo de este estudio fue evaluar en qué medida la información aportada por 11 parámetros nutricionales para 47 especies forestales puede verse simplificada a través de la aplicación de ACP, y así, comprobar si el uso de esta técnica multivariante facilita el análisis comparativo de la calidad nutricional entre distintas formaciones forestales que a menudo funcionan como sistemas silvopastorales en Galicia. 


\section{Material y Métodos}

\subsection{Especies vegetales y análisis químicos}

A partir de datos recopilados de diversos trabajos (González-Hernández y SilvaPando, 1999; González-Hernández et al., 2000), se elaboró una matriz numérica representada por 11 parámetros nutricionales para 47 especies vegetales presentes en robledales, pinares y matorrales gallegos (Tab. 1). Dichos datos describen los contenidos en $\mathrm{P}, \mathrm{Ca}, \mathrm{Mg}, \mathrm{K}$, las fracciones de fibra (FAD, lignina, celulosa, sílice), el porcentaje de materia seca (MS), la digestibilidad in vitro (DMO) y el contenido en proteína bruta $(\mathrm{PB})$. En éstos, para el análisis de los elementos minerales se realizó una digestión Kjeldhal, estimándose el $\mathrm{P}$ con un analizador de flujo continuo TRAACS-800 y el Ca, Mg y K con espectrofotometría de absorción atómica. Las fracciones de fibra se obtuvieron según la técnica de análisis detergente secuencial

Tabla 1. Asociaciones vegetales que componen el estudio y relación de especies ${ }^{\mathrm{a}}$ más frecuentes.

\begin{tabular}{ll}
\hline Asociaciones vegetales & \multicolumn{1}{c}{ Especies analizadas más representativas } \\
\hline Blechno spicant & AGCA, AGCU, ANAM, ANNE, ASPH, BEAL, BRSY, CARE, \\
-Quercetum roboris (robledales) & CASA, CYSC, CYST, DIPU, ERAR, FASY, FRAL, GEFL, HEHE, \\
Rusco aculeati & HOMO, ILAQ, LANO, LOPE, LUHE, POER, POOD, PSEU, PTAQ, \\
-Quercetum roboris (robledales) & PYCO, QROB, ROPS, RUAC, RUBS, TESC, VAMY \\
Vaccinio myrtilli & \\
-Quercetum roboris (robledales) & \\
Linario triornithophorae & \\
-Quercetum pyrenaicae (robledales) & \\
\hline
\end{tabular}

Omphalodo nitidae

-Fagetum sylvaticae (hayedos)

FASY, HOMO, ANNE, AGCU, BRSY, VAMY OMPH, ILAQ, HEHE, ERAR, PYCO

Ulici europaei-Ericetum cinerae (tojales, brezales y pinares)

AGCA, AGCU, ASPH, BRSY, CAVU, DACN, ERAR, ERCI, ERCN, ERMA, ERUM, HALA, PSEU, ULEU, ULMI

Gentiano pneumonanthes-Ericetum mackaianae (pinares, brezales)

DACN, ERCN, ERCI, ULGA, PSEU, ASPH, AGCA, AGCU, POER, PTRI, ERUM, FAAL, ULEU, ERMA, CAVU, TESC, RUBS

Genistello tridentatae

-Ericetum aragonensis (brezales)

ERAU, PTRI, HALA, AGCA, AGCU, HAUM, ASPH

\footnotetext{
a Especies: AGCA: Agrostis capillaris, AGCU: Agrostis curtissii, ANAM: Anthoxanthum amarum, ANNE: Anemone nemorosa, ASPH: Asphodelus albus, BEAL: Betula alba, BRSY: Brachypodium sylvaticum, CARE: Carex remota, CASA: Castanea sativa, CAVU: Calluna vulgaris, CYSC: Cytisus scoparius, CYST: Cytisus striatus, DACN: Daboecia cantabrica, DIPU: Digitalis purpurea, ERAR: Erica arborea, ERAU: Erica australis, ERCI: Erica ciliaris, ERCN: Erica cinerea, ERMA: Erica mackaiana, ERUM: Erica umbellata, EUGL: Eucalyptus globulus, FASY: Fagus sylvatica, FRAL: Frangula alnus, GEFL: Genista florida, PTRI: Pterospartum tridentatum, HALA: Halimium lasianthum, HAUM: Halimiun umbellatum, HEHE: Hedera helix, HOMO: Holcus mollis, ILAQ: Ilex aquifolium, LANO: Laurus nobilis, LOPE: Lonicera peryclimenum, LUHE: Luzula henriquessii, OMPH: Omphalodes lusitanica, POER: Potentilla erecta, POOD: Polygonatum odoratum, PSEU: Pseudarrhenatherum longifolium, PTAQ: Pteridium aquilinum, PYCO: Pyrus cordata, QROB: Quercus robur, ROBS: Robinia pseudacacia, RUAC: Ruscus aculeatus, RUBS: Rubus sp., TESC: Teucrium scorodonia, ULEU: Ulex europaeus, ULGA: Ulex gallii, ULMI: Ulex minor, VAMY: Vaccinium myrtillus.
} 
de Göering and Van Soest (1970), empleando un sistema Fibertec DOSIFIBER 4000599 (Selecta). La digestibilidad in vitro se determinó según la metodología de Tilley and Terry (1963) (citada en González-Hernández y Silva-Pando, 1999). El contenido proteico se estimó según el método micro-Kjeldahl. Más detalles adicionales sobre los procedimientos de extracción y las técnicas de análisis de laboratorio aplicadas se pueden consultar en González-Hernández y Silva-Pando (1999) y González-Hernández et al., (2000).

\subsection{Análisis estadístico}

El ACP se realizó para 11 parámetros nutricionales en 47 especies (matriz de datos 11 x 47). Sólo se seleccionaron componentes principales con valores propios (eigenvalues) iguales o superiores a 1, ya que, valores inferiores, representan menos varianza que la variable original (con varianza igual a 1) y son de poca utilidad (Johnson and Wichern, 2002). Adicionalmente, se tuvieron en cuenta otros criterios tales como la salida gráfica del test scree, el porcentaje mínimo de varianza acumulada y el criterio de interpretabilidad. Para identificar a los parámetros nutricionales que mejor definen la variabilidad absorbida por cada componente principal extraído se procedió a su rotación ortogonal Varimax, al permitir ésta identificar con mayor nitidez a los parámetros que más contribuyen a la descripción de cada componente (i.e. factores con mayor peso en un componente dado). Para la realización de los ACP se utilizó el software STATGRAPHICS Plus Version 5.0.

\section{Resultados y discusión}

El ACP original se realizó para un conjunto de 11 parámetros nutricionales en 47 especies forestales con el objetivo de ordenar la información nutricional de especies presentes en robledales, pinares y matorrales gallegos, y evaluar las posibles diferencias o similitudes entre estas formaciones. La finalidad del ACP fue obtener un número reducido de combinaciones lineales de los parámetros nutricionales que explicasen la mayor parte de la variabilidad de los datos e investigar las relaciones nutricionales entre las especies estudiadas. Tras el primer análisis de una matriz de datos 11 x 47, se decidió retirar el parámetro sílice al trasladar éste un porcentaje significativo de la varianza a un tercer componente principal, e interferir en la selección de una más sencilla representación bidimensional de los datos. De este modo, se realizó un segundo ACP con una matriz 10 x 47 que cumplió mejor los criterios de interpretabilidad, obteniéndose también una mejor salida gráfica. En la Tab. 2 se presenta la fortaleza de la relación lineal entre las diferentes variables medidas a través del análisis del coeficiente de correlación de Pearson para la matriz final de datos.

El ACP extrajo dos componentes principales de variación basándose en los criterios de eigenvalues $\geq 1$. La combinación de ambos componentes explicó un $66 \%$ de la varianza total de los datos. Tras la rotación ortogonal Varimax de los dos com- 
Tabla 2. Matriz de correlación de Pearson entre los parámetros nutricionales estudiados. Fibra Ácido Detergente (FAD), lignina, celulosa, proteína bruta (PB), digestibilidad in vitro (DMO), materia seca $(\mathrm{MS})$, fósforo $(\mathrm{P})$, calcio $(\mathrm{Ca})$, magnesio $(\mathrm{Mg})$ y potasio $(\mathrm{K})$.

\begin{tabular}{|c|c|c|c|c|c|c|c|c|c|}
\hline & $\mathbf{P}$ & $\mathrm{Ca}$ & $\mathrm{Mg}$ & $\mathrm{K}$ & FAD & Lignina & Celulosa & MS & $\mathrm{DMO}$ \\
\hline $\mathrm{Ca}$ & -0.148 & & & & & & & & \\
\hline $\mathrm{Mg}$ & 0.166 & 0.278 & & & & & & & \\
\hline K & $0.542 *$ & 0.009 & $0.424^{*}$ & & & & & & \\
\hline FAD & $-0.425^{*}$ & -0.233 & $-0.349 *$ & $-0.513 *$ & & & & & \\
\hline Lignina & -0.058 & $0.373^{*}$ & 0.193 & 0.111 & 0.214 & & & & \\
\hline Celulosa & $-0.426^{*}$ & $-0.380^{*}$ & $-0.496^{*}$ & $-0.629 *$ & $0.878^{*}$ & -0.175 & & & \\
\hline MS & $-0.423 *$ & -0.161 & $-0.435^{*}$ & $-0.850 *$ & $0.598^{*}$ & -0.118 & $0.727 *$ & & \\
\hline DMO & $0.434^{*}$ & 0.140 & 0.221 & $0.718^{*}$ & $-0.764^{*}$ & 0.047 & $-0.761 *$ & $-0.797^{*}$ & \\
\hline PB & $0.439 *$ & 0.033 & 0.228 & $0.601 *$ & $-0.496 *$ & 0.063 & $-0.569^{*}$ & $-0.642 *$ & $0.680^{*}$ \\
\hline
\end{tabular}

* indica correlaciones estadísticamente significativas al nivel de confianza del $95 \%$.

ponentes retenidos, aquellos parámetros que presentaron coeficientes $\geq 0.70$ para un componente y $<0.70$ para el otro, se consideraron significativos y explicativos del mismo. Se decidió seleccionar aquellas variables con coeficientes $\geq 0.70$, al ser una cifra que representó bien el punto de corte entre los valores más altos y más bajos de dichos coeficientes, y además, haberse considerado este valor anteriormente en otros trabajos (Eni et al., 2012). Utilizando tal criterio, seis parámetros (DMO, $\mathrm{K}, \mathrm{PB}, \mathrm{ADF}$, celulosa y MS) explicaron la mayor variabilidad de los datos en el primer componente, y sólo dos (Ca y lignina) representaron al segundo (Tab. 3).

Las relaciones entre los parámetros nutricionales quedaron bien diferenciadas en la salida del diagrama de ordenación del ACP (Fig. 1).

Tabla 3. Coeficientes ${ }^{\mathrm{a}}$ (peso) de los parámetros nutricionales tras la rotación Varimax de los dos primeros componentes.

\begin{tabular}{|c|c|c|}
\hline \multirow{2}{*}{ Variables } & \multicolumn{2}{|c|}{ Componentes Principales } \\
\cline { 2 - 3 } & 1 & 2 \\
\hline $\mathrm{P}$ & 0.6586 & -0.2619 \\
$\mathrm{Ma}$ & 0.0443 & $\mathbf{0 . 8 2 9 2}$ \\
$\mathrm{Mg}$ & 0.4005 & 0.5141 \\
$\mathrm{~K}$ & $\mathbf{0 . 8 4 9 7}$ & 0.0769 \\
FAD & $\mathbf{0 . 8 1 8 0}$ & -0.0898 \\
Lignina & -0.0619 & $\mathbf{0 . 7 2 6 0}$ \\
Celulosa & $\mathbf{0 . 8 3 3 8}$ & -0.3950 \\
MS & $\mathbf{- 0 . 8 6 8 5}$ & -0.2049 \\
DMO & $\mathbf{0 . 8 8 8 5}$ & 0.0909 \\
PB & $\mathbf{0 . 7 6 6 8}$ & 0.0056 \\
\hline
\end{tabular}

${ }^{\text {a }}$ Variables en negrita con coeficientes superiores a 0.70 en valor absoluto se consideran significativas en cada componente. 


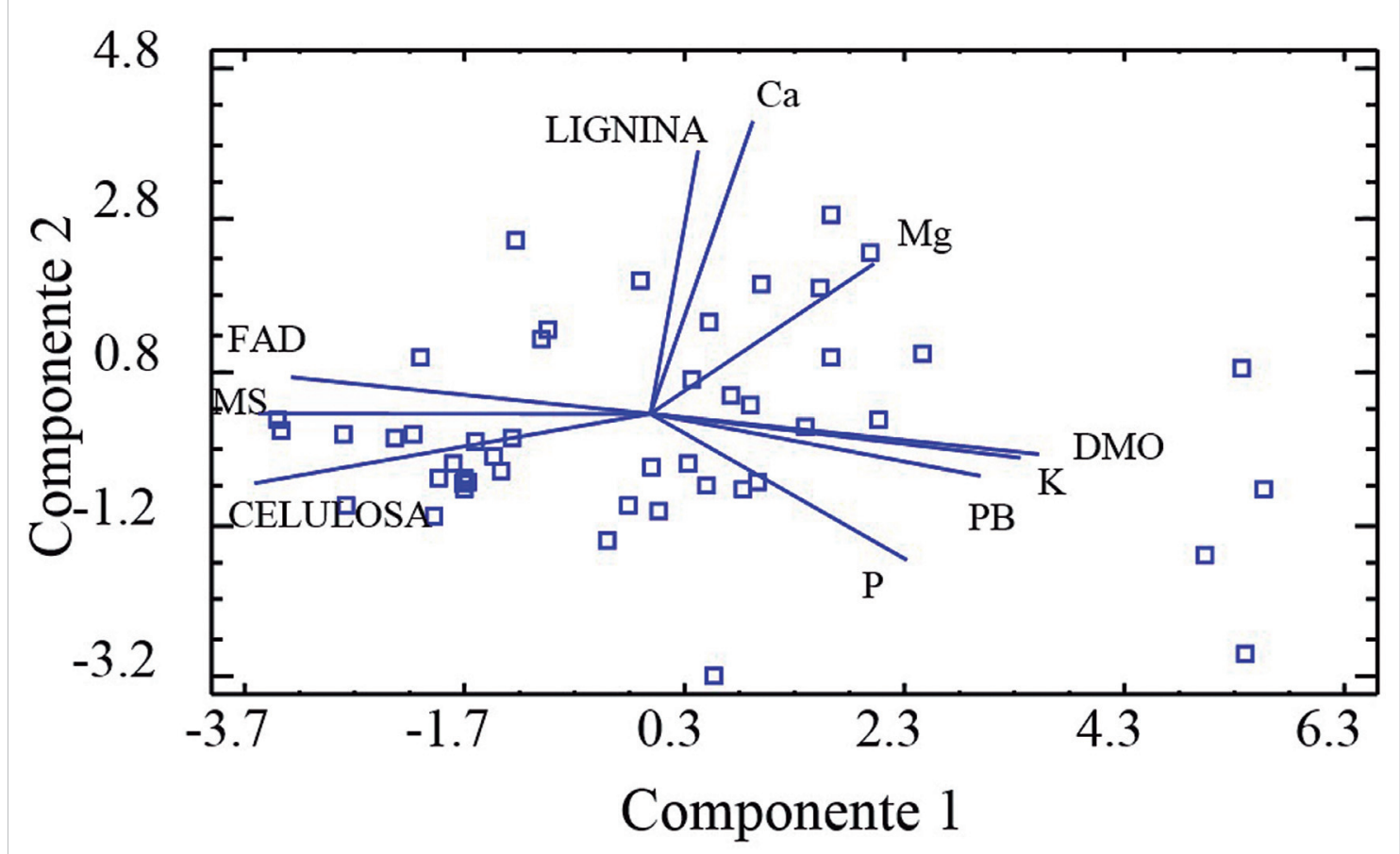

Figura 1. Gráfica bidimensional del ACP de la relación entre los parámetros nutricionales con la magnitud (representatividad o peso) y la dirección espacial para cada uno de ellos. FAD: Fibra Ácido Detergente, lignina, celulosa, PB: proteína bruta, DMO: digestibilidad in vitro, MS: materia seca, fósforo $(\mathrm{P})$, calcio $(\mathrm{Ca})$, magnesio $(\mathrm{Mg})$ y potasio $(\mathrm{K})$.

E1 50\% de la varianza explicada por el componente 1 se basó principalmente en los parámetros digestibilidad y contenido en $\mathrm{K}$, hacia el lado positivo del eje, y al contenido en fibra (sobre todo celulosa) y materia seca, hacia el lado negativo. El componente 2 representó a un mayor contenido de lignina y Ca en las especies (explicando un $16 \%$ de la varianza). La polaridad marcada por dichos parámetros nutricionales se manifiesta en la matriz de correlación de Pearson (Tab. 2). Aunque bien es sabido que la PB es esencial a la hora de interpretar el valor nutricional en las dietas, la diferencia entre especies con respecto a este parámetro, fue menos acusada que la digestibilidad y el $\mathrm{K}$, explicando, por tanto dicha variable, un menor porcentaje de la variabilidad de los datos (Tab. 3).

La representación gráfica de los dos componentes principales retenidos agrupó a especies con un mayor contenido en FAD, MS y celulosa, y por tanto, con una menor calidad nutritiva, hacia el extremo más negativo del primer componente (Fig. 2). Estas especies representan a las familias Ericaceae (Erica australis, E. mackaiana, E. cinerea, E. umbellata, E. ciliaris, Calluna vulgaris, Daboecia cantabrica), Poaceae (Agrostis curtissii, Pseudarrhenatherum longifolium), y Cistaceae (Halimium umbellatum, H. lasianthum), así como a leguminosas arbustivas (Ulex gallii, U. minor, U. europaeus, Cytisus striatus y C. scoparius), y caracterizan a algunas de las más típicas comunidades de pinares, brezales y tojales existentes en Galicia (Gentiano pneumonanthes-Ericetum mackaianae, Genistello tridentatae- 
Ericetum aragonensis, Ulici europaei-Ericetum cinerae), ya reconocidas como de calidad nutritiva inferior al compararlas con la del sotobosque bajo arbolado caducifolio (González-Hernández y Silva-Pando, 1999).

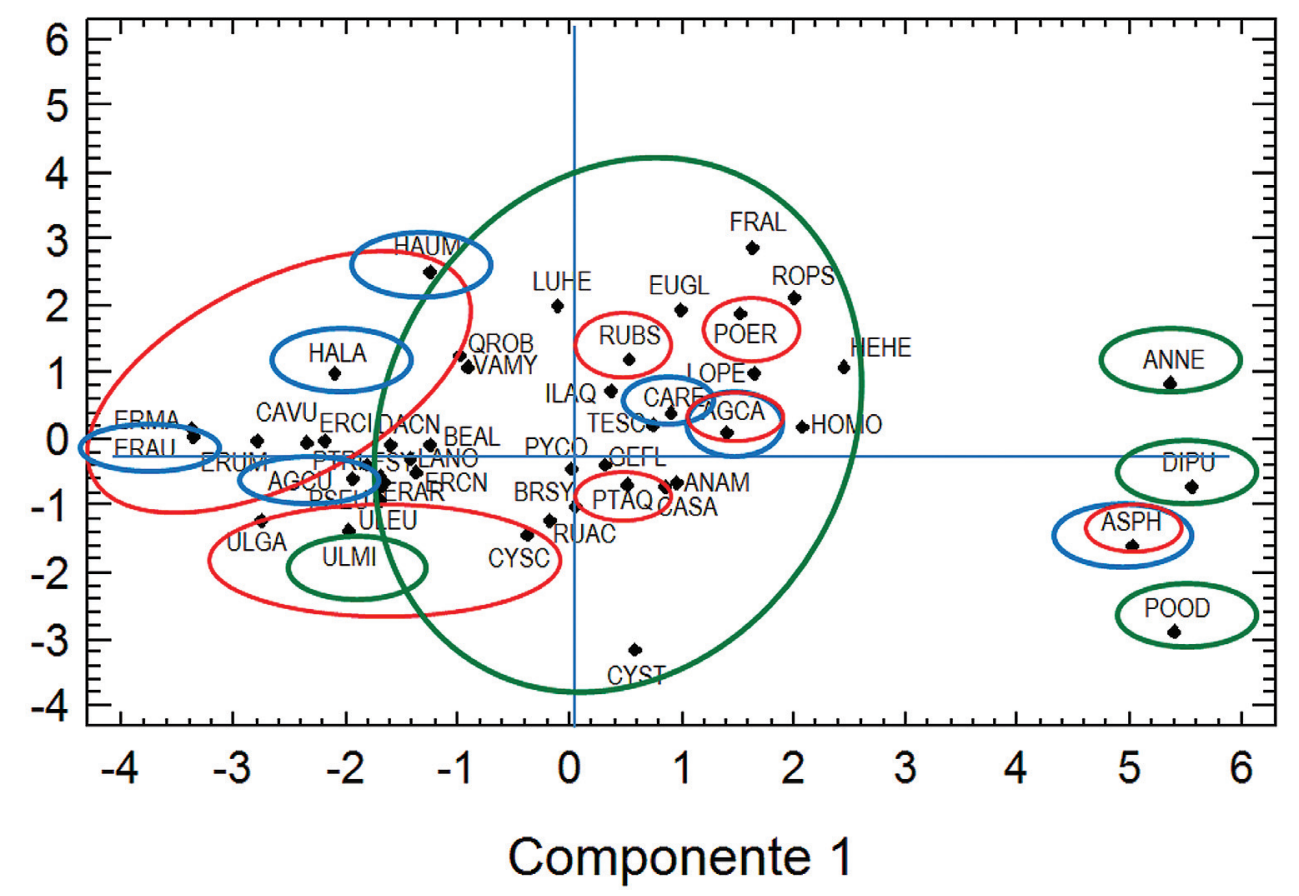

Figura 2. Ordenación gráfica bidimensional de las especiesa en el ACP (véase Figura 1). Se englobaron en verde (๑) las especies más comunes de robledales, en rojo $(\bullet)$ las propias de tojales, brezales y pinares (Gentiano pneumonanthes-Ericetum mackaianae y Ulici europaei-Ericetum cinerae) y en azul (๑) los brezales de Erica australis (Genistello tridentatae-Ericetum aragonensis).

\begin{abstract}
a Especies: AGCA: Agrostis capillaris, AGCU: Agrostis curtissii, ANAM: Anthoxanthum amarum, ANNE: Anemone nemorosa, ASPH: Asphodelus albus, BEAL: Betula alba, BRSY: Brachypodium sylvaticum, CARE: Carex remota, CASA: Castanea sativa, CAVU: Calluna vulgaris, CYSC: Cytisus scoparius, CYST: Cytisus striatus, DACN: Daboecia cantabrica, DIPU: Digitalis purpurea, ERAR: Erica arborea, ERAU: Erica australis, ERCI: Erica ciliaris, ERCN: Erica cinerea, ERMA: Erica mackaiana, ERUM: Erica umbellata, EUGL: Eucalyptus globulus, FASY: Fagus sylvatica, FRAL: Frangula alnus, GEFL: Genista florida, PTRI: Pterospartum tridentatum, HALA: Halimium lasianthum, HAUM: Halimiun umbellatum, HEHE: Hedera helix, HOMO: Holcus mollis, ILAQ: Ilex aquifolium, LANO: Laurus nobilis, LOPE: Lonicera peryclimenum, LUHE: Luzula henriquessii, OMPH: Omphalodes lusitanica, POER: Potentilla erecta, POOD: Polygonatum odoratum, PSEU: Pseudarrhenatherum longifolium, PTAQ: Pteridium aquilinum, PYCO: Pyrus cordata, QROB: Quercus robur, ROBS: Robinia pseudacacia, RUAC: Ruscus aculeatus, RUBS: Rubus sp., TESC: Teucrium scorodonia, ULEU: Ulex europaeus, ULGA: Ulex gallii, ULMI: Ulex minor, VAMY: Vaccinium myrtillus.
\end{abstract}

Retamas como Cytisus scoparius, y otras ericáceas como Erica arborea y Vaccinium myrtillus, forman parte habitualmente de comunidades de robledales en distintos grados de sucesión vegetal, y aparecen representadas en la gráfica del ACP junto a Quercus robur, Laurus nobilis y Ruscus aculeatus, situándose hacia el origen de los dos ejes. Estas especies representan bien a los robledales termófilo galaico-portugueses septentrionales acidófilos de la serie Rusco aculeati-Quercetum roboris (Rivas Martínez, 2011). En el lado positivo del eje 1, coincidiendo con una mayor representación de los parámetros K, PB y digestibilidad aparecen agrupadas 
especies tales como Ilex aquifolium, Teucrium scorodonia, Lonicera periclymenum, Hedera hiberna, Frangula alnus, y poáceas más digestibles como Agrostis capillaris, Anthosantum amarum y Holcus mollis que forman parte del sotobosque de comunidades de robledal de carácter menos mediterráneo y pertenecientes a las serie Vaccinio myrtilli-Quercetum roboris y Blechno spicant-Quercetum roboris (Rivas Martínez, 2011) (Fig. 2). Hacia el extremo más positivo de este eje se localizaron especies herbáceas indicadoras de robledales poco antropizados como Anemone nemorosa y Polygonatum odoratum, además de otras de carácter ruderal, como Digitalis purpurea o abundantes en zonas que han sufrido incendios como $\mathrm{As}$ phodelus albus. Dichos robledales ya se señalaron como las comunidades forestales gallegas con calidad nutritiva más alta en González-Hernández y Silva-Pando (1999).

En la Tab. 4 se pueden observar los valores medios de los distintos parámetros nutricionales para las formaciones forestales del presente estudio. Los robledales se presentan como la formación forestal cuyo sotobosque ofrece las más altas calidades nutritivas (basadas en un menor contenido en fibras, y mayores valores en contenido mineral, PB y digestibilidad); aunque destaquen en cuanto al contenido proteico también otras formaciones compuestas mayoritariamente por leguminosas, como piornales y tojales.

Tabla 4. Valores nutricionales para las formaciones forestales estudiadas expresados como valor medio \pm desviación estándar del conjunto de las especies que las componen (FAD: fibra ácido detergente, MS: materia seca, DMO: digestibilidad de la materia orgánica, PB: proteína bruta).

\begin{tabular}{|c|c|c|c|c|c|c|c|}
\hline $\begin{array}{c}\text { Parámetro } \\
\text { nutricional } \\
(\%)\end{array}$ & Brezales & Eucaliptales & Pinares & Robledales & Tojales & Piornales & Hayedos \\
\hline FAD & $68.0 \pm 6.5$ & $62.6 \pm 12.3$ & $67.1 \pm 7.3$ & $56.5 \pm 10.9$ & $64.0 \pm 6.4$ & $62.3 \pm 4.9$ & $59.5 \pm 8.6$ \\
Lignina & $17.6 \pm 2.8$ & $14.9 \pm 4.3$ & $15.7 \pm 3.6$ & $16.6 \pm 4.5$ & $16.1 \pm 3.1$ & $17.4 \pm 2.7$ & $17.5 \pm 2.7$ \\
Celulosa & $46.0 \pm 5.6$ & $43.9 \pm 10.6$ & $46.9 \pm 6.5$ & $35.6 \pm 8.5$ & $45.6 \pm 7.4$ & $41.8 \pm 5.6$ & $37.3 \pm 7.6$ \\
Sílice & $4.4 \pm 4.1$ & $3.9 \pm 3.9$ & $4.4 \pm 3.5$ & $4.2 \pm 4.4$ & $2.3 \pm 2.7$ & $3.1 \pm 2.4$ & $4.6 \pm 3.2$ \\
MS & $47.8 \pm 9.1$ & $40.9 \pm 10.6$ & $42.6 \pm 8.8$ & $34.7 \pm 10.0$ & $39.2 \pm 9.3$ & $44.0 \pm 8.9$ & $34.5 \pm 10.7$ \\
$\mathrm{DMO}$ & $28.1 \pm 9.6$ & $29.1 \pm 15.3$ & $27.0 \pm 9.2$ & $42.7 \pm 14.3$ & $42.6 \pm 18.1$ & $44.5 \pm 14.8$ & $44.4 \pm 14.9$ \\
$\mathrm{~PB}$ & $6.8 \pm 1.0$ & $7.8 \pm 2.4$ & $8.3 \pm 3.4$ & $9.8 \pm 3.5$ & $9.3 \pm 2.3$ & $12.6 \pm 3.9$ & $9.9 \pm 4.0$ \\
$\mathrm{P}$ & $0.13 \pm 0.07$ & $0.14 \pm 0.14$ & $0.15 \pm 0.14$ & $0.18 \pm 0.12$ & $0.15 \pm 0.15$ & $0.17 \pm 0.02$ & $0.24 \pm 0.12$ \\
$\mathrm{Ca}$ & $0.32 \pm 0.21$ & $0.24 \pm 0.14$ & $0.23 \pm 0.18$ & $0.33 \pm 0.29$ & $0.20 \pm 0.13$ & $0.13 \pm 0.04$ & $0.51 \pm 0.67$ \\
$\mathrm{Mg}$ & $0.07 \pm 0.05$ & $0.06 \pm 0.04$ & $0.05 \pm 0.03$ & $0.08 \pm 0.09$ & $0.05 \pm 0.03$ & $0.09 \pm 0.11$ & $0.08 \pm 0.04$ \\
$\mathrm{~K}$ & $0.23 \pm 0.17$ & $0.27 \pm 0.19$ & $0.27 \pm 0.24$ & $0.50 \pm 0.37$ & $0.26 \pm 0.15$ & $0.32 \pm 0.27$ & $0.54 \pm 0.38$ \\
$\mathrm{Ca}: \mathrm{P}$ & 2.56 & 1.72 & 1.49 & 1.79 & 1.38 & 0.79 & 2.10 \\
$\mathrm{~K}:(\mathrm{Ca}+\mathrm{Mg})$ & 0.59 & 0.86 & 0.99 & 1.23 & 1.04 & 1.42 & 0.92 \\
\hline
\end{tabular}

En Galicia, los pinares en su mayoría se asientan en terrenos donde anteriormente residían robledales o bien en terrenos convertidos de matorral. Por ello, algunas especies que los componen se distribuyeron a lo largo de una amplia sección 
del eje 1. Por ejemplo, Rubus sp., Potentilla erecta, Pteridium aquilinum y Agrostis capillaris (y otras poáceas) es frecuente que aparezcan en robledales algo degradados y también en pinares provenientes de antiguos robledales aprovechados para madera. Esto está representado en la salida gráfica por las intersecciones que engloban círculos de diferente color (Fig. 2). Los hayedos están representados por un estado sucesional asociado a comunidades de robledal al no haberse incluido en este estudio especies clave diferenciadoras de los mismos.

El componente 2, representado por el eje de ordenadas, y con un peso significativo de los parámetros nutricionales Ca y lignina en su lado positivo (Fig. 2), separó gradualmente a lo largo del mismo a plantas con contenidos más elevados en Ca como Frangula alnus, Hedera hiberna, Anemone nemorosa, Vaccinium myrtillus, Rubus sp., y Teucrium scorodonia. Otras leguminosas arbustivas y también gramíneas se situaron gradualmente hacia zonas menos positivas de este eje. El Ca y la fracción indigestible lignina, son parámetros relacionados en ocasiones con problemas de absorción de otros minerales o con tetania, y con un aporte menor de energía en las dietas, respectivamente, y por tanto, este segundo componente ejemplificaría, en oposición al primero, a un componente vinculado a desórdenes nutricionales. En cualquier caso, dicho componente contribuyó, con apenas un 16\% de absorción de varianza, de forma bastante limitada a agrupar o separar a las especies por formaciones forestales.

Aunque las concentraciones de $\mathrm{Ca}$ en muchas de las especies que componen la vegetación forestal gallega se encuentran en el rango óptimo para cubrir las necesidades de animales en pastoreo (González-Hernández et al., 2000), concentraciones altas de $\mathrm{K}$ asociadas a bajas concentraciones de $\mathrm{Ca}$ y $\mathrm{Mg}$ pueden resultar en ratios de $\mathrm{K}:(\mathrm{Ca}+\mathrm{Mg})$ mayores de 2.2 e inducir tetania, enfermedad metabólica común en rumiantes que se alimentan de gramíneas de crecimiento rápido con bajo contenido en Ca y/o Mg (Butler, 1963). Sin embargo, a pesar de las bajas concentraciones de $\mathrm{Mg}$ en las principales especies de la vegetación forestal gallega (González-Hernández et al., 2000), este mismo estudio reveló valores del cociente $\mathrm{K}:(\mathrm{Ca}+\mathrm{Mg})$ por lo general, inferiores a 2.2 que fueron indicativos de un buen equilibrio entre estos minerales y, por tanto, de un bajo riesgo de tetania (González-Hernández et al., 2000). Los valores medios en el componente mineral obtenidos para cada formación forestal en el presente estudio (Tab. 4) corroboran estos resultados, e indican valores por debajo de 2.2 en el parámetro $\mathrm{K}:(\mathrm{Ca}+\mathrm{Mg})$ para todas las formaciones estudiadas.

Muchos estudios de nutrición animal apuntan también la necesidad de un buen ratio Ca:P, resultando óptimos aquellos cocientes con valores entre 1 y 2 (Hejcman et al., 2016). En base a este rango, las formaciones del presente estudio tienen, en general, valores óptimos, excepto los brezales, que presentan valores sensiblemente superiores a 2 (Tab. 4). Se sabe que, valores superiores, que supongan un exceso de Ca considerable en relación al $\mathrm{P}$ en la dieta, pueden reducir la absorción de este último, al igual que la de otros minerales ( $\mathrm{Mg}, \mathrm{Mn}, \mathrm{Zn}$, etc), resultando ello en menores crecimientos y peores índices de mineralización ósea (NRC, 1994; 1998). Por tanto, los brezales gallegos, con valores altos en este ratio, podrían ver acentua- 
dos sus ya valores bajos en $\mathrm{P}$ y provocar, en algunas situaciones de pastoreo, problemas asociados a la escasez de este elemento mineral en la dieta si no se corrige con suplementos adicionales.

Dado que los ratio entre diferentes elementos minerales pueden ser indicativos de la disponibilidad de los mismos, y por tanto afectar a la salud del animal en pastoreo, se realizó un ACP adicional incluyendo los cocientes $\mathrm{Ca}: \mathrm{P}$ y K:(Ca+Mg). Los principales parámetros que definieron al componente principal 1 fueron comunes a los del ACP inicial; es decir, el componente 1 ordenó a las especies en base a su mayor o menor digestibilidad, contenido proteico y $\mathrm{K}$ (definido el componente 1 por $\mathrm{DMO}, \mathrm{K}, \mathrm{PB}, \mathrm{ADF}$, celulosa y $\mathrm{MS}$ ), aunque se sumaron con coeficientes significativos los ratio $\mathrm{K}:(\mathrm{Ca}+\mathrm{Mg})$ al primer componente, y el ratio $\mathrm{Ca}: \mathrm{P}$ al segundo (desplazando, en este caso, en importancia a la lignina) (Tab. 5, Fig. 3).

Tabla 5. Coeficientes ${ }^{\mathrm{a}}$ (peso) de los parámetros nutricionales tras la rotación Varimax de los dos primeros componentes.

\begin{tabular}{|c|c|c|}
\hline \multirow{2}{*}{ Variables } & \multicolumn{2}{|c|}{ Componentes Principales } \\
\cline { 2 - 3 } & 1 & 2 \\
\hline $\mathrm{P}$ & 0.5096 & -0.1089 \\
$\mathrm{Mg}$ & 0.0325 & $\mathbf{0 . 8 9 4 6}$ \\
$\mathrm{K}$ & 0.4079 & 0.5218 \\
$\mathrm{FAD}$ & $\mathbf{0 . 8 9 6 4}$ & 0.0066 \\
Lignina & $\mathbf{- 0 . 7 0 9 2}$ & -0.1774 \\
Celulosa & 0.04709 & 0.5860 \\
Sílice & $\mathbf{- 0 . 7 9 8 3}$ & -0.4218 \\
$\mathrm{MS}$ & 0.02025 & 0.0212 \\
$\mathrm{DMO}$ & $\mathbf{- 0 . 9 1 5 9}$ & -0.1342 \\
$\mathrm{~PB}$ & $\mathbf{0 . 8 7 8 1}$ & 0.0429 \\
$\mathrm{Ca}: \mathrm{P}$ & $\mathbf{0 . 8 0 0 5}$ & -0.0659 \\
$\mathrm{~K}: \mathrm{CaMg}$ & -0.1489 & $\mathbf{0 . 7 5 1 6}$ \\
$\mathrm{N}: \mathrm{P}$ & $\mathbf{0 . 7 0 2 3}$ & -0.4493 \\
& 0.2322 & 0.0706 \\
\hline
\end{tabular}

${ }^{\text {a }}$ Variables en negrita con coeficientes superiores a 0.70 en valor absoluto se consideran significativas en cada componente.

El resultado de dicho ACP indicó que cuatro componentes principales cumplían los criterios para su extracción, acumulando un $80 \%$ de la variabilidad de los datos. Sin embargo, la representación gráfica de los dos primeros componentes acumuló un 59\% de la variabilidad de los datos, siendo un porcentaje inferior al $66 \%$ explicado por los dos primeros componentes del ACP previo. Esto se tradujo en una ordenación de las especies en el espacio bidimensional que no aportó información adicional relevante en cuanto a agrupaciones de especies diferenciadoras de comunidades forestales (Fig. 4). 


\section{Plot of Component Weights}

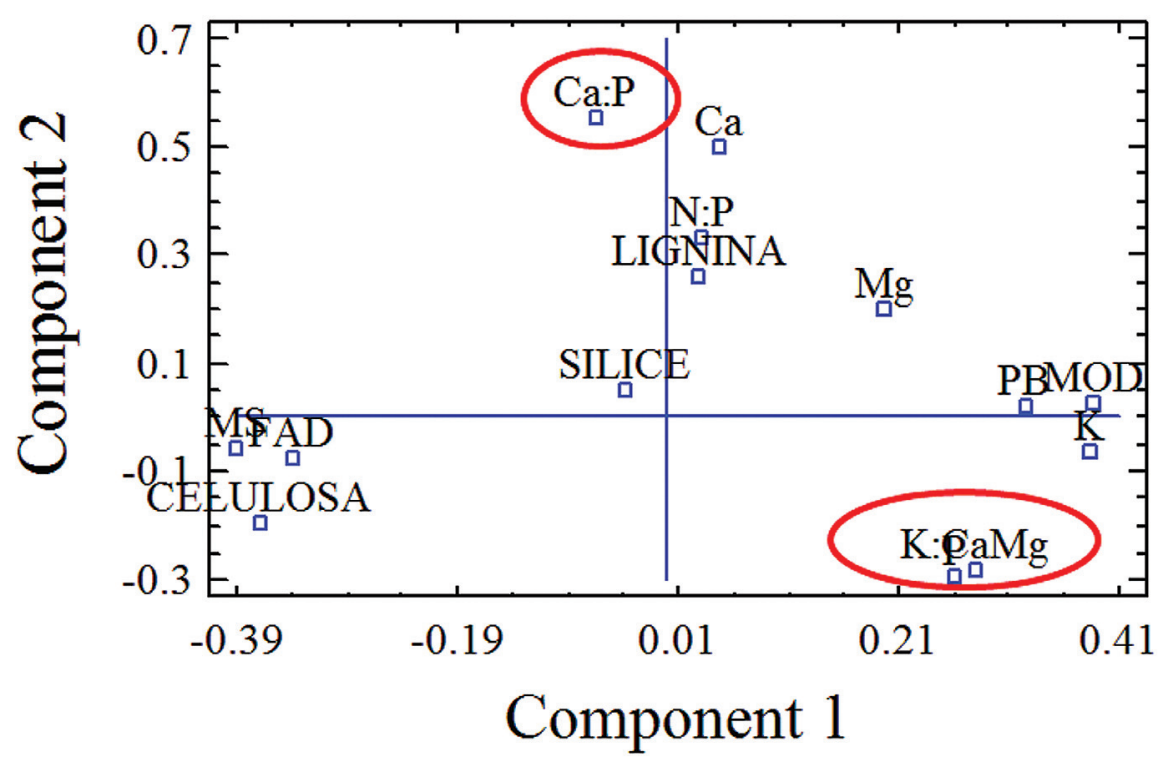

Figura 3. Gráfica bidimensional del ACP de la relación entre los parámetros nutricionales y los ratios $\mathrm{Ca}: \mathrm{P}$ y K:(Ca+Mg) que indica la magnitud (representatividad o peso) y la dirección espacial para cada uno de ellos. FAD: Fibra Ácido Detergente, lignina, celulosa, PB: proteína bruta, DMO: digestibilidad in vitro, MS: materia seca, fósforo $(\mathrm{P})$, calcio $(\mathrm{Ca})$, magnesio $(\mathrm{Mg})$ y potasio $(\mathrm{K})$.

Cabe resaltar, sin embargo, que algunas especies cambiaron su ordenación inicial y podría atribuírsele, entonces, una cierta utilidad a la inclusión de los ratios $\mathrm{K}:(\mathrm{Ca}+\mathrm{Mg})$ y $\mathrm{Ca}: \mathrm{P}$, aportando su consideración información adicional con respecto a un gradiente que ordena a las especies en base a su capacidad de aportar riesgo de tetania (Fig. 4). Por ejemplo, aquellas especies ubicadas hacia el lado positivo del eje 1 y hacia el negativo del eje 2 (Asphodelus albus, Digitalis purpurea y Polygonatum odoratum) tienen valores más elevados en el ratio $\mathrm{K}:(\mathrm{Ca}+\mathrm{Mg})$, menores concentraciones de $\mathrm{Ca}$ e inferiores ratios $\mathrm{Ca}: \mathrm{P}$, y por tanto, podría deducirse que una mayor proporción de las mismas en las comunidades vegetales podría ir en detrimento de una buena absorción del $\mathrm{Mg}$ (altas concentraciones de $\mathrm{K}$ o bajas de $\mathrm{P}$ interfieren en una buena absorción del $\mathrm{Mg}$ ).

Los resultados de nuestro estudio corroboran los de otros autores en cuanto a la utilidad del ACP para simplificar la interpretación de las similitudes o diferencias entre un número elevado de especies vegetales definidas por numerosos parámetros nutricionales (Hejcman et al., 2014; Hejcmanová et al., 2014; GonzálezHernández et al., 2016; Hejcman et al., 2016;). Los ACP efectuados han permitido establecer gradientes de mayor o menor calidad nutricional entre las especies y mostrar agrupaciones coherentes de varias comunidades forestales de forma bastante definida. La inclusión de los ratio Ca:P y K:(Ca+Mg), y su peso o significación en los dos primeros componentes, ha incorporado un gradiente adicional orien- 


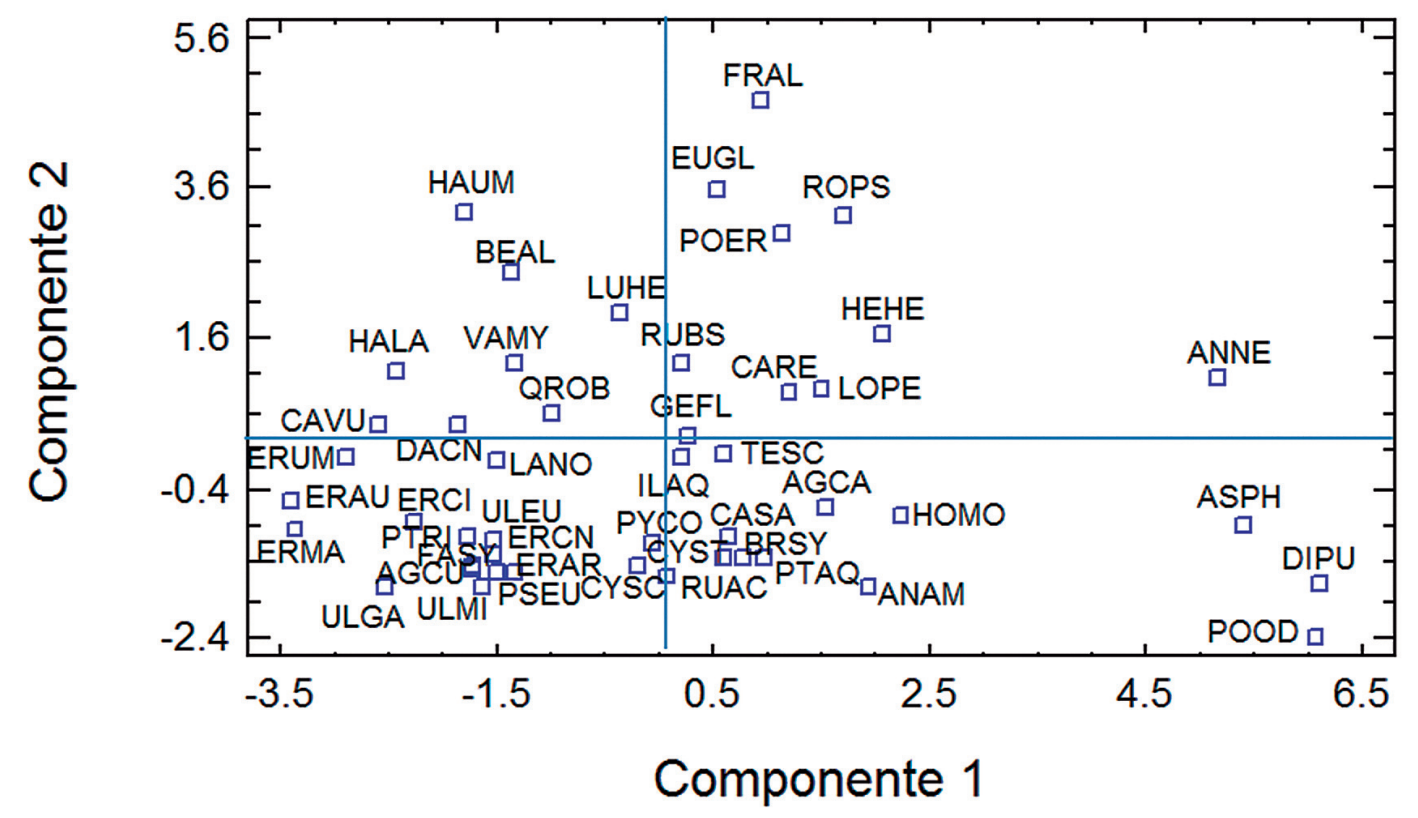

Figura 4. Ordenación gráfica bidimensional (dos primeros componentes) de las especies en el ACP que incluye a los ratios Ca:P y K:(Ca+Mg). AGCA: Agrostis capillaris, AGCU: Agrostis curtissii, ANAM: Anthoxanthum amarum, ANNE: Anemone nemorosa, ASPH: Asphodelus albus, BEAL: Betula alba, BRSY: Brachypodium sylvaticum, CARE: Carex remota, CASA: Castanea sativa, CAVU: Calluna vulgaris, CYSC: Cytisus scoparius, CYST: Cytisus striatus, DACN: Daboecia cantabrica, DIPU: Digitalis purpurea, ERAR: Erica arborea, ERAU: Erica australis, ERCI: Erica ciliaris, ERCN: Erica cinerea, ERMA: Erica mackaiana, ERUM: Erica umbellata, EUGL: Eucalyptus globulus, FASY: Fagus sylvatica, FRAL: Frangula alnus, GEFL: Genista florida, PTRI: Pterospartum tridentatum, HALA: Halimium lasianthum, HAUM: Halimiun umbellatum, HEHE: Hedera helix, HOMO: Holcus mollis, ILAQ: Ilex aquifolium, LANO: Laurus nobilis, LOPE: Lonicera peryclimenum, LUHE: Luzula henriquessii, POER: Potentilla erecta, POOD: Polygonatum odoratum, PSEU: Pseudarrhenatherum longifolium, PTAQ: Pteridium aquilinum, PYCO: Pyrus cordata, QROB: Quercus robur, ROBS: Robinia pseudacacia, RUAC: Ruscus aculeatus, RUBS: Rubus sp., TESC: Teucrium scorodonia, ULEU: Ulex europaeus, ULGA: Ulex gallii, ULMI: Ulex minor, VAMY: Vaccinium myrtillus.

tativo para distinguir aquellas especies con mayor probabilidad de provocar posibles desórdenes nutricionales como la hipomagnesemia.

\section{Conclusiones}

La descripción de la calidad nutritiva de 47 especies forestales, que han caracterizado los recursos silvopastorales del noroeste de la Península Ibérica en estudios previos (González-Hernández y Silva-Pando, 1999; González-Hernández et al., 2000), ha sido sometida a un ACP. Se revela la efectividad de cuatro parámetros a la hora de caracterizar la calidad nutritiva del conjunto de especies que componen el estudio y de las formaciones forestales consideradas; estos fueron su contenido 
en $\mathrm{K}$ y en celulosa, además de su digestibilidad, y cantidad de MS. Adicionalmente, otros 3 parámetros: $\mathrm{Ca}, \mathrm{Ca}: \mathrm{P}, \mathrm{y} \mathrm{K}:(\mathrm{Ca}+\mathrm{Mg})$ han ordenado a las especies según su grado de contribución a desórdenes nutricionales como la hipomagnesemia. La aplicación de esta técnica multivariante ha permitido reducir 14 parámetros (11 variables y 3 ratios) a casi la mitad, sin perderse información relevante y simplificando así la interpretación de los datos.

\section{Bibliografía}

Butler, E.J., 1963. The mineral element content of spring pasture in relation to the occurrence of grass tetany and hypomagnesaemia in dairy cows. J. Agric. Sci. 60, 329-340. https://doi.org/10.1017/S0021859600011916

Eni, D.D., Iwara, A.I., Offiong, R.A., 2012. Analysis of soil-vegetation interrelationships in a south-southern secondary forest of Nigeria. Intl. J. For. Res. 2012, 1-8. https://doi.org /10.1155/2012/469326

Göering, H.K., Van Soest, P.J., 1970. Forage fiber analyses (Apparatus, Reagents, Procedures, and Some Applications). Agriculture Handbook 379, Agricultural Research Service, USDA, pp. 1-19.

González-Hernández, M.P., Silva-Pando, F.J., 1999. Nutritional attributes of understory plants known as components of deer diets. J. Range Manage. 52 (2), 132-138. https:// doi.org/10.2307/4003506

González-Hernández, M.P., Silva-Pando, F.J., Mosquera-Losada, M.R., Rigueiro, A., 2000. Contenido mineral de especies componentes del monte gallego (NW Espa-a). Importancia en la gestión de ecosistemas pascícolas. In: Actas de la XL Reunión Ibérica de Pastos y Forrajes. Xunta de Galicia.

González-Hernández, M.P., Mosquera-Losada, M.P., Ferreiro-Domínguez, N., Rigueiro, A., 2016. A multivariate approach in the analysis of the nutritional quality of woody pastures in NW Spain. In: Casasús, I., Lombardi, G., (eds.), Mountain pastures and livestock farming facing uncertainty: environmental, technical and socio-economic challenges. Options méditerranéennes, Series A: Mediterranean Seminars, 116, 281286.

Hejcman, M., Hejcmanová, P., Stejskalová, M, Pavlu, V., 2014. Nutritive value of winter-collected annual twigs of main European woody species, mistletoe and ivy and its possible consequences for winter foddering of livestock in prehistory. The Holocene 24(6), 659 -667. https://doi.org/10.1177/0959683614526904

Hejcman, M., Hejcmanová, P., Pavlu, V., Thorhallsdottir, A.G., 2016. Forage quality of leaf fodder from the main woody species in Iceland and its potential use for livestock in the past and present. Grass and Forage Sci 71(4), 649-658. https://doi.org/10.1111/gfs. 12224

Hejcmanová, P., Stejskalová, M., Hejcman, M., 2014. Forage quality of leaf-fodder from the main broad-leaved woody species and its possible consequences for the Holocene development of forest vegetation in Central Europe. Veget. Hist. Archaeobot. 23, 607 -613. https://doi.org/10.1007/s00334-013-0414-2

Johnson, R.A., Wichern, D.E., 2002. Applied Multivariate Statistical analysis. Prentice Hall, NJ, USA. 
NRC (1994). Nutrient requirements of poultry. 9th revised ed. National Research Council, National Academy Press, Washington D.C.

NRC (1998). Nutrient requirements of swine. 10th revised ed. National Research Council, National Academy Press, Washington D.C.

Rivas Martínez, S., 2011. Mapa de series, geoseries y geopermaseries de vegetación de Espa-a. [Memoria del mapa de vegetación potencial de Espa-a] PARTE II. Itinera geobotanica 18(1), 5-800.

Van Soest, P.J., 1982. Nutritional ecology of the ruminant. Ruminant metabolism, nutritional strategies, the cellulolytic fermentation and the chemistry of forages and plant fibers. Cornell University, New York. 